\title{
From Petri Nets to Polynomials: Modeling, Algorithms, and Complexity (Abstract) (Invited Talk)
}

\author{
Ernst W. Mayr \\ Institut für Informatik, \\ Technische Universität München \\ Boltzmannstr. 3, 85748 Garching, Germany \\ mayr@in.tum.de
}

\begin{abstract}
Petri nets are a widely-used model for parallel and distributed systems of concurrent systems using common resources. They admit a precise algebraic formalization as vector addition or vector replacement systems. If one considers symmetric VRS's, it turns out that they are equivalent to finitely presented commutative semigroups or to binomial ideals in a multivariate ring over $\mathbb{Q}$.

We outline and survey the interaction between these domains of computational algebra, system modeling and verification, and, in particular, complexity theory. While many of the fundamental computational problems in these areas turn out to be very complex (i.e., EXPSPACEcomplete or even worse, we also present some new results concerning better complexity for restricted subclasses of the problems.
\end{abstract}

